\title{
New progress in aggregation-induced emission research
}

\author{
LI Zhen \\ Department of Chemistry, Wuhan University, Wuhan 430072, China
}

Received March 2, 2010; accepted June 9, 2010

The most fascinating aspect of scientific research is the constant deciphering of mysterious puzzles and the continuous transformation of our mode of thinking. While textbook knowledge is the foundation of human wisdom, some traditional concepts can later prove to be imperfect or even incorrect owing to limitations of the observations and understanding of the involved natural phenomena in particular periods of history. The once popular "geocentric theory", for example, was totally wrong and hampered scientists' exploration of the universe, and the "vitality of the doctrine" badly hindered advancement in organic chemistry research. Innovations are needed to break the restraints of such traditional concepts.

The area of organic solids research focuses on investigations of molecular functional materials with peculiar optical, electronic and magnetic properties. In this area, a generally accepted concept is that chromophore aggregation always leads to luminescence quenching. This phenomenon of aggregation-caused quenching (ACQ) is termed the "concentration-quenching effect" in the textbook Molecular Fluorescence. Researchers are working on the development of organic light-emitting diodes (OLEDs), in which luminescent molecules are used as a solid film, one of the aggregation forms. Scientists have tried hard to suppress the aggregate formation of luminescent molecules through different approaches, with the aim of mitigating the ACQ effect and achieving OLEDs with high efficiencies.

In 2001, Tang's group discovered a phenomenon exactly opposite to the ACQ effect. The researchers found that luminogen aggregation played a constructive, instead of destructive, role in the light-emitting process; a series of silole molecules, such as hexaphenylsilole (HPS) and methylpentaphenylsilole (MPPS; Figure 1), were found to be nonluminescent in the solution state but emissive in the aggregated state with luminescent quantum yields three orders of

email: lizhen@whu.edu.cn magnitude higher than those of the diluted solutions. They coined the term "aggregation-induced emission" (AIE) for this novel phenomenon. This discovery overturned the general belief of the ACQ effect and instantly attracted dynamic and passionate interest from academic and industrial circles. As a result, it opened up the research field of AIE materials and their applications [1]. Using AIE luminogens as light-emitting layers, researchers have fabricated an OLED with an external quantum efficiency as high as $8 \%$, well approaching the theoretical limit for electroluminescence devices based on organic singlet emitters.

Based on their systemic studies, Tang's group concluded that the AIE phenomenon is mainly caused by the restriction of intramolecular motion in the aggregate state [2]. Under the guidance of the mechanistic understanding, Tang's group and other scientists developed a wide variety of AIE systems. In the folding structures of biopolymers such as proteins and deoxyribonucleic acids, there are hydrophobic pockets that can be considered hydrophobic binding sites. Once AIE molecules bind to these hydrophobic regions, their intramolecular vibrational and rotational motions should be restricted, thus leading to an induced strong fluorescence. In other words, an off/on switch of light emission may be realized and utilized for probing the presence of biopolymers and their folding and unfolding (or denaturing) processes as well as the accompanying conformational changes. With this in mind, Tang's group has designed many different types of amphipathic AIE molecules as biosensors to probe biopolymers with high selectivity and sensitivity. Moreover, the off/on process can be readily discerned by the naked eye from the dark background. This kind of fluorescence turn-on biosensor is superior to turn-off counterparts and is less likely to generate false-positive signals. In addition, by modifying the molecular structures of AIE luminogens, different biopolymers can be detected selectively $[2,3]$.

Recently, Tang's group successfully extended the applications of AIE molecules to the field of cell imaging. Researchers reported their use of AIE luminogen 1 (Figure 1) 

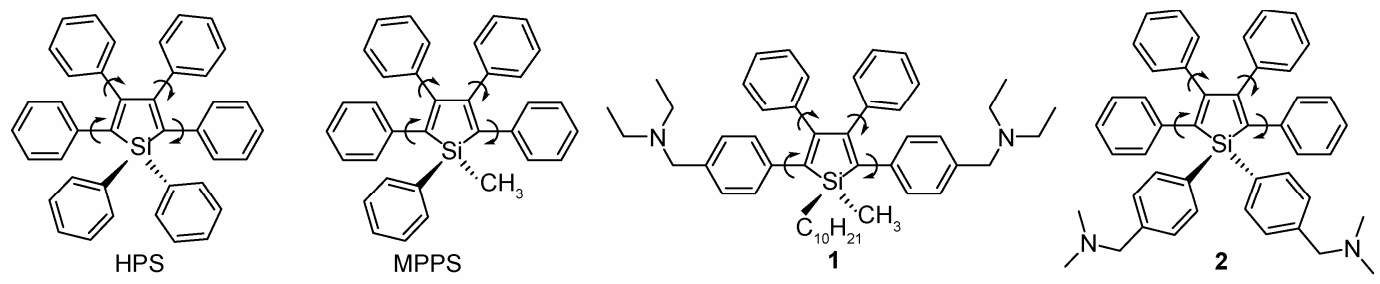

Figure 1 Structures of some silole molecules.

as a visualization reagent [4]. The work has three noteworthy features: (1) the cells can be clearly imaged in an exposure time as short as $1 \mathrm{~s}$, while it takes $5 \mathrm{~s}$ using the generally used commercial dye CellTracker ${ }^{\mathrm{TM}}$ Green CMFDA to do the same job; (2) the area stained by $\mathbf{1}$ is concentrated in the cytoplasmic region of the cells, whereas CMFDA stains the entire cells, making it difficult to distinguish different parts of the cells; and (3) $\mathbf{1}$ is nontoxic and cytocompatible without interfering with the metabolisms of living cells, whereas other generally used labels are inherently poisonous (e.g., quantum dots) or of concern in terms of toxicity; for example, the surfactants used in the fabrications of polymer and silica nanoparticles such as sodium dodecyl sulfate can trigger cytolysis processes even at a concentration as low as $0.0005 \%$ [4]. Cell imaging with high sensitivity, selectivity and biocompatibility offers a direct visualization tool for detecting biological macromolecules and monitoring biological events under real, living conditions and is of great importance to bioscience and biotechnology. The significant results of Tang's group provide a new approach to, and stimulate further development of, novel fluorescent dyes for cell imaging. Recently, Tang et al. has summarized the utilization of AIE molecules, so far, in the field of biosensing in their review paper [5].

Tang's group utilized AIE molecules for determining the critical micelle concentration (CMC) [6]. As mentioned above, the AIE effect originates from the restriction of intramolecular rotations of the peripheral phenyl groups attached to the conjugated cores in the aggregates. In an aqueous solution of a lipophilic AIE molecule such as HPS or 2 (Figure 1), a faint fluorescence is expected in the presence of a minute amount of surfactant. When the amount of surfactant approaches or exceeds the CMC, the surfactant molecules will self-assemble into micelles. Driven by hydrophobic interaction, the lipophilic AIE molecules will enter and aggregate in the hydrophobic cores of the micelles to provide amplified fluorescence, which can be recorded and even seen by the naked eye. Their experimental results are consistent with data obtained employing classical techniques, but their method is much simpler and can be realized simply with a normal ultraviolet lamp at low cost without using sophisticated equipment. Thus, Tang et al. have proposed a convenient method for CMC determination. Since micelles are ubiquitous and important owing to their multiple functionalities such as media for emulsion polymerization, templates for nanostructure synthesis, and carriers for controllable drug delivery, Tang's method for CMC determination is anticipated to be used in an array of technological applications.

The two examples discussed above $[4,6]$, together with the other exciting results achieved by Tang's group [2,5], clearly demonstrate that by tuning their molecular structures, the AIE luminogens can be applied to different research fields. Attracted by the abnormality of the AIE phenomenon and the unorthodox behaviors of the AIE luminogens, many research groups have been engaged in the study of AIE. It is envisioned that the collective efforts of these enthusiastic scientists will push the AIE research field to an even higher climax.

1 Luo J, Xie Z, Lam J W Y, et al. Aggregation-induced emission of 1methyl-1,2,3,4,5-pentaphenylsilole. Chem Commun, 2001, 1740-1741

2 Hong Y, Lam J W Y, Tang B Z. Aggregation-induced emission: Phenomenon, mechanism and applications. Chem Commun, 2009, 4332-4353

3 Li Z, Dong Y, Lam J W Y, et al. Functionalized siloles: Versatile synthesis, aggregation-induced emission, and sensory and device applications. Adv Funct Mater, 2009, 19: 905-917

4 Yu Y, Hong Y, Feng C, et al. Synthesis of an AIE-active fluorogen and its application in cell imaging. Sci China Ser B-Chem, 2009, 52: 15-19

5 Yan J, Qin A, Sun J, et al. Application of AIE-active molecules in biosensing (in Chinese). Chinese Sci Bull (Chinese Ver), 2010, 55: 1206-1213

6 Tang L, Jin J, Zhang S, et al. Detection of the critical micelle concentration of cationic and anionic surfactants based on aggregationinduced emission property of hexaphenylsilole derivatives. Sci China Ser B-Chem, 2009, 52: 755-759 